\title{
Dispositif de formation pour le développement de la compétence à l'oral de futurs enseignants dans un contexte d'approche-programme : quelles sont les pratiques déclarées efficaces?
}

\author{
SYLVIE VIOLA \\ Université du Québec à Montréal \\ GENEVIÈVE MESSIER \\ Université du Québec à Montréal \\ CHRISTIAN DUMAIS \\ Université du Québec à Trois-Rivières \\ HÉLÈNE MEUNIER \\ Université du Québec à Montréal
}

\section{Résumé}

La compétence langagière est au centre de la profession enseignante (Plessis-Bélair, 2006). Que ce soit à l'écrit ou à l'oral, l'enseignant doit être un modèle pour ses élèves. Pour cela, il doit avoir un niveau de compétence suffisamment élevé. Malgré son importance, le développement de la compétence langagière demeure une difficulté omniprésente chez les futurs enseignants, notamment en ce qui concerne le volet oral (Tremblay et Mottet, 2012). À partir de ce constat, le programme d'éducation préscolaire et d'enseignement primaire de l'Université du Québec à Montréal a proposé à ses étudiants un dispositif de formation comprenant diverses interventions visant le développement de la compétence à l'oral. Après trois ans d'expérimentation de ces pratiques, il apparaissait pertinent de connaitre lesquelles étaient considérées comme étant les plus efficaces. Pour ce faire, un questionnaire a d'abord été distribué à 563 étudiants du programme. Par la suite, des entretiens de groupe ont été réalisés. Les résultats issus de l'analyse des entrevues permettent de faire des liens avec les résultats obtenus à la suite de la passation du questionnaire et d'interpréter ces résultats à la lumière des principes de l'approche-programme.

\section{Mot-clés (5)}

Compétence à l'oral, dispositif de formation, objets de l'oral, approche-programme, pratiques déclarées

\section{Introduction}

Le développement de la compétence à l'oral, qui consiste à être en mesure d'utiliser adéquatement la langue d'enseignement à l'oral dans les divers contextes liés à la profession enseignante, doit être au cœur des interventions des programmes 
de formation à l'enseignement. En effet, l'oral occupe une place centrale en classe puisque la grande partie du travail de l'enseignant est constituée d'interactions, d'échanges, d'explications, de questionnements et d'écoute. Malgré les recherches qui ont porté sur cette dimension importante de la compétence professionnelle « Communiquer clairement et correctement dans la langue d'enseignement, à l'oral et à l'écrit, dans les divers contextes liés à la profession enseignante » (Gouvernement du Québec, 2001a), cette préoccupation est récente dans le contexte d'approcheprogramme. Cette approche se définit comme

[une démarche] d'organisation et de fonctionnement constructive et évolutive visant le développement optimal des apprenants qui composent cette organisation. Elle est possible grâce à une certaine forme de leadership au sein du programme et à la mise en œuvre de stratégies de communication, de concertation, de coordination et d'engagement provenant des différents acteurs concernés par cette organisation. (Viola, 2011, p. 2)

L'approche-programme a comme enjeu de proposer un agencement cohérent de stratégies didactiques et pédagogiques fondé sur la concertation des acteurs qui composent les programmes. En effet, ce contexte, contrairement à celui de l'approche-cours, mise fortement sur les répartitions des objets d'apprentissage et sur le développement progressif des compétences. À cet égard, nous présenterons dans ce texte la problématique entourant le développement de la compétence à l'oral des futurs enseignants du programme d'éducation préscolaire et d'enseignement primaire (ÉPEP) à l'Université du Québec à Montréal (UQAM). Nous expliquerons par la suite le concept de dispositif de formation qui nous a amenés à circonscrire et à organiser les interventions présentes dans le programme. Nous expliquerons le choix de notre méthodologie de recherche axée sur la théorisation enracinée qui nous a permis d'avoir accès aux pratiques déclarées d'étudiants des quatre années de formation du programme ÉPEP. Nous présenterons ensuite les résultats obtenus et nous en discuterons à la lumière de travaux de recherche issus de la didactique de l'oral et de la formation professionnelle. Enfin, quelques recommandations seront suggérées dans le but de faciliter le développement de la compétence à l'oral dans le contexte de la mise en œuvre de l'approche-programme.

\section{Problématique}

La compétence à l'oral est au cœur du métier enseignant (Dumais, Messier, Viola et Meunier, 2013). En plus d'être un modèle à l'oral pour ses élèves, l'enseignant utilise constamment l'oral pour enseigner et interagir avec eux. Or, au cours des dernières années, c'est plutôt la compétence à l'écrit qui a été considérée comme prioritaire dans les programmes de formation à l'enseignement (Dumais et Goyette, 2012; Messier, Dumais et Viola, 2012). En 2001, les États généraux sur la situation et l'avenir de la langue française au Québec dénonçaient le fait que la moitié des futurs enseignants québécois ne possédait pas une maitrise de la langue française suffisante pour exercer la fonction d'enseignant (Gouvernement du Québec, 2001b). À partir de ce constat, plusieurs mesures ont été mises en place dans la plupart des 
universités pour aider les étudiants à maitriser la compétence à l'écrit : test de certification, ateliers individuels et collectifs, site internet, épreuve unique TECFÉE (Test de certification en français écrit pour l'enseignement), etc., laissant en partie pour compte la compétence à l'oral. Pourtant, la compétence «Communiquer clairement et correctement dans la langue d'enseignement, à l'oral et à l'écrit, dans les divers contextes liés à la profession enseignante », qui fait partie du référentiel de compétences professionnelles de la profession enseignante (Gouvernement du Québec, 2001a), concerne autant l'oral que l'écrit. Comment expliquer le peu de préoccupation pour la compétence à l'oral dans les programmes de formation initiale? À l'UQAM, nous pouvons expliquer cela entre autres par le fait que les lacunes en ce qui concerne cette compétence sont rarement sanctionnées chez les étudiants lors des stages ou des cours, alors que c'est le cas pour la compétence à l'écrit. De plus, il semble plus difficile d'intervenir sur la compétence à l'oral à l'université étant donné l'augmentation constante du nombre d'étudiants dans les groupes (Viola, Messier, Dumais et Meunier, 2015). À cet égard, il faut aussi mentionner qu'il existe présentement une évaluation très variée de l'oral dans les cours puisque les objets de l'oral sont peu connus par l'ensemble des responsables de cours (Dumais, 2015). À l'UQAM, la seule épreuve d'évaluation de la compétence à l'oral pour tous les étudiants en enseignement a lieu à la fin de la première année de formation. Seuls les étudiants qui échouent cette passation sont dans l'obligation de suivre des cours d'appoint portant sur différents volets de l'oral (volet linguistique [voix et langue], communicationnel et discursif). À ce propos, dans le programme ÉPEP de l'UQAM, le taux moyen de réussite des étudiants pour les années 2010 à 2013 est de $65 \%$. Ainsi, plusieurs étudiants n'auront pas de cours sur l'oral à suivre, sauf le seul cours obligatoire qui est en fait un cours de didactique de l'oral. Même si une partie de ce cours propose aux étudiants d'effectuer un portrait du locuteur (Dumais, 2015), ceci nous apparait nettement insuffisant pour former les étudiants au développement de leur propre compétence. En effet, une compétence se développe sur une longue période et nécessite plus d'une activité pour permettre aux étudiants de s'améliorer (Desjardins et Dezutter, 2009). Bref, en ce qui concerne la compétence à l'oral, il y a peu d'interventions pédagogiques explicites, concertées et progressives pour encadrer le développement de cette compétence chez les étudiants. Ainsi, l'approcheprogramme était une occasion idéale de mettre en place de telles interventions. En effet, cette approche définie précédemment est une

démarche d'ingénierie pédagogique visant à assurer la cohérence et l'harmonisation au sein et entre les différentes composantes d'un programme d'études afin de favoriser l'intégration des apprentissages chez l'étudiant tout au long de son parcours d'étude (Basque et Rogozan, 2013).

Elle permet de répartir à la fois les objets de 1'oral (25 objets, dont la prononciation, l'intonation, le regard, etc., qui sont issus d'une recherche de Préfontaine, Lebrun et Nachbauer [1998] sur la compétence à l'oral des futurs enseignants) sur les quatre années de formation et les interventions pédagogiques indispensables au développement de cette compétence. Ainsi, au programme ÉPEP 
de l'UQAM, plusieurs initiatives ont été mises en place depuis 2010 afin d'aider les étudiants en ce sens. En effet, l'équipe programme s'est concertée pour élaborer une grille d'autoévaluation et de coévaluation de l'oral qui a été diffusée aux différents acteurs du programme (Dumais et al., 2013). À partir de cette grille, il a été possible de former les étudiants aux objets de l'oral et de planifier une situation d'apprentissage et d'évaluation (SAÉ) par année de formation, soit quatre au total (Viola et al., 2015). Ces situations ont été complétées par d'autres interventions (des formations spécifiques sur certains objets de l'oral par exemple) afin de proposer un dispositif de formation. Il nous est donc apparu essentiel de vérifier la valeur pédagogique des interventions de ce dispositif de formation en demandant aux étudiants ce qu'ils en pensaient. Ceci nous a amenés à formuler la question de recherche suivante : quelles interventions formelles contenues dans ce dispositif de formation sont les plus favorables au développement des compétences à l'oral des étudiants en formation initiale au programme ÉPEP?

\section{Cadre théorique}

Au cours des quatre années de leur baccalauréat, les étudiants du programme ÉPEP de l'UQAM ont été exposés à différentes interventions pédagogiques pour favoriser le développement de leur compétence à l'oral. Le dispositif de formation que nous proposons ici regroupe toutes ces interventions. Cet agencement ne pouvait se réaliser sans une vision concertée du programme par ses différents acteurs. Cette démarche d'ingénierie pédagogique appelée « approche-programme » vise justement à assurer la cohérence et l'harmonie entre les différentes composantes d'un programme (Viola, Dumais et Messier, 2012). En s'inspirant du modèle de Paquette (2002), trois composantes de l'approche-programme ont permis d'éclairer le cadre général de cette recherche, soit la composante des cibles d'apprentissage, la composante pédagogique et la composante médiatique. Les cibles d'apprentissage font référence aux connaissances et aux compétences à développer par les étudiants. Dans le contexte de la présente recherche, comme nous l'avons mentionné précédemment, il s'agit des objets de l'oral et de la compétence 2 du référentiel de compétences professionnelles de la profession enseignante. La composante pédagogique concerne les approches pédagogiques privilégiées par le programme et leur amalgame à l'échelle du programme, ce que nous avons appelé le dispositif de formation. Enfin, la composante médiatique fait référence à l'ensemble du matériel didactique utilisé. Dans le cadre de cette recherche, les étudiants pouvaient avoir accès à des documents vidéo, des sites web et des documents textuels. Le tableau 1 illustre les différentes interventions du dispositif de formation tout au long du cheminement de quatre ans. Par exemple, en première année de formation, le dispositif contient deux interventions de type diagnostique et de remédiation (DR), deux interventions de type transversal et progressif (TP) et deux interventions de type non interventionniste (NI). Lors de cette première année, les étudiants sont exposés à un plus grand nombre d'interventions, car le programme les prépare à l'examen de l'oral qui aura lieu au mois de mai de cette même année. En deuxième année, le programme poursuit ce qui a été entamé en première année en mettant en application 
la grille d'autoévaluation dans la SAÉ l'art de conter et dans les stages. Les cours d'appoint (LIN1611 et les autres) viennent compléter les interventions de la deuxième

Tableau 1

Dispositif de formation et types d'interventions pédagogiques des quatre années du baccalauréat

\begin{tabular}{|c|c|c|c|}
\hline $\begin{array}{c}\text { Libre et facultatif } \\
\text { (LF) }\end{array}$ & $\begin{array}{c}\text { Diagnostique et de } \\
\text { remédiation } \\
\text { (DR) }\end{array}$ & $\begin{array}{c}\text { Non } \\
\text { interventionniste } \\
\text { (NI) }\end{array}$ & $\begin{array}{c}\text { Transversal et } \\
\text { progressif } \\
\text { (TP) }\end{array}$ \\
\hline
\end{tabular}

\begin{tabular}{|c|c|c|c|c|c|c|c|c|}
\hline \multirow[b]{2}{*}{ 这 } & & \multicolumn{2}{|c|}{ DDM1650 } & DDD $1210^{3}$ & & & & \\
\hline & $\begin{array}{l}\text { Portrait du } \\
\text { Locuteur }\end{array}$ & $\begin{array}{r}\text { Prés } \\
\text { de } 1 \\
\text { d } \\
\text { éva }\end{array}$ & $\begin{array}{l}\text { ation } \\
\text { rille } \\
\text { to- } \\
\text { tion }\end{array}$ & $\begin{array}{l}\text { SAÉ 1: les } \\
\text { réseaux } \\
\text { conceptuels }\end{array}$ & $\begin{array}{c}\text { Application } \\
\text { de la grille } \\
\text { dans le } \\
\text { cours }\end{array}$ & & $\begin{array}{l}\text { lication } \\
\text { la grille } \\
\text { as les } \\
\text { tages }\end{array}$ & $\begin{array}{l}\text { Épreuve } \\
\text { d'évalua- } \\
\text { tion de } \\
\text { l'oral }\end{array}$ \\
\hline \multirow{3}{*}{ 离 } & DDM26 & & \multicolumn{2}{|c|}{$\begin{array}{c}\text { LIN1016 } \\
(1017-1018-1019- \\
1020)\end{array}$} & \multicolumn{2}{|c|}{ DDD 2100} & \multicolumn{2}{|c|}{ DDM2600 } \\
\hline & $\begin{array}{r}\text { SAÉ } 2: 1^{\prime} \\
\text { conte }\end{array}$ & rt de & & \multicolumn{2}{|c|}{$\begin{array}{l}\text { Pratique de l'art } \\
\text { de conter et } \\
\text { application de la } \\
\text { grille }\end{array}$} & \multicolumn{2}{|c|}{$\begin{array}{l}\text { Application de la } \\
\text { grille dans les } \\
\text { stages }\end{array}$} \\
\hline & \multicolumn{3}{|c|}{ DDM3600 } & \multicolumn{2}{|c|}{ DDD2210 } & \multicolumn{3}{|c|}{ DDM3551 } \\
\hline 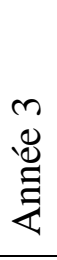 & \multicolumn{3}{|c|}{$\begin{array}{l}\text { Application de la grille } \\
\text { dans les stages }\end{array}$} & \multicolumn{2}{|c|}{$\begin{array}{l}\text { Application de la grille } \\
\text { dans le cours }\end{array}$} & \multicolumn{3}{|c|}{ SAÉ 3 : le débat éthique } \\
\hline \multirow[b]{2}{*}{ 离 } & \multicolumn{3}{|c|}{ DDM4650 } & \multicolumn{2}{|c|}{ DDM410X } & \multicolumn{3}{|c|}{ DDM4600 } \\
\hline & \multicolumn{3}{|c|}{$\begin{array}{l}\text { SAÉ } 4: \text { les interactions } \\
\text { professionnelles }\end{array}$} & \multicolumn{2}{|c|}{$\begin{array}{l}\text { Application de la grille } \\
\text { dans le cours }\end{array}$} & \multicolumn{3}{|c|}{$\begin{array}{l}\text { Application de la grille } \\
\text { dans les stages }\end{array}$} \\
\hline
\end{tabular}

${ }^{1}$ DDL : cours du département de didactique des langues

${ }^{2}$ DDM : cours du département de didactique, domaine des stages

${ }^{3}$ DDD : cours du département de didactique

${ }^{4}$ MAT : cours du département de mathématiques

${ }^{5}$ Xoral : test oral

${ }^{6} \mathrm{LIN}$ : cours du département de linguistique 
année. Enfin, pour effectuer le stage 3 (en troisième année), les étudiants doivent avoir réussi les cours ou l'examen. C'est la raison pour laquelle il n'existe plus d'interventions de type diagnostique et de remédiation. En quatrième année, le programme participe au développement progressif (TP) de la compétence à l'oral à l'aide d'une SAÉ portant sur les interactions professionnelles et encourage les étudiants à utiliser la grille d'autoévaluation et de coévaluation dans les cours et dans les stages. Des interventions de type libre et facultatif (LF) sont offertes tout au long de la formation de quatre ans.

\subsection{Les dispositifs de formation}

Les dispositifs de formation peuvent être définis selon Weisser (2010, p. 292) comme «une articulation d'éléments hétérogènes, matériels et symboliques ». Ils représentent l'ensemble des moyens mis en œuvre afin d'atteindre un but déterminé. Selon Caron (2007), il faut ajouter la dimension humaine à la liste des éléments invoqués. Il mentionne aussi, en citant Peeters et Charlier (1999), que les dispositifs constituent un espace de potentialisation ouvert dans lequel quelque chose peut se produire en fonction des acteurs concernés (Paquelin et Choplin, 2003). Il y a donc, dans le concept de dispositif, une ouverture possible qui ferait en sorte que ceux-ci puissent être bénéfiques aux apprenants à des niveaux variables. Le dispositif de formation proposé ici comporte toutes nos interventions pédagogiques et a comme objectif général le développement de la compétence à l'oral. Il poursuit aussi des objectifs spécifiques qui démontrent bien le concept d'hétérogénéité des éléments qui compose ce dispositif en classant les interventions en quatre catégories selon le type d'encadrement privilégié : libre et facultatif, diagnostique et de remédiation, non interventionniste, et transversal et progressif. De plus, le dispositif fait appel à l'oral comme objet ou comme médium (Dolz et Schneuwly, 1998; Lafontaine, 2001). Le matériel requis pour un dispositif varie en fonction des objectifs poursuivis.

\subsection{L'oral médium et l'oral objet}

Des recherches menées en Suisse et au Québec (Dolz et Schneuwly, 1998; Lafontaine, 2001) ont permis de déterminer deux statuts de l'oral. Ce dernier peut être considéré comme un oral médium lorsqu'il est utilisé comme moyen pour communiquer de l'information sans qu'il y ait de retour spécifique sur la façon de s'exprimer. Il s'agit d'un oral utilitaire. Par exemple, dans le contexte de l'enseignement universitaire, l'oral médium est utilisé lorsqu'un formateur utilise l'oral pour transmettre de l'information aux étudiants sans qu'un retour soit effectué sur la façon de s'exprimer. Le deuxième statut est celui d'oral objet. Il s'agit d'un oral pour lequel il est possible de fixer des objectifs d'apprentissage à atteindre et des compétences à faire développer (Lafontaine, 2001). Il s'agit donc d'un oral enseigné, c'est-à-dire qu'un retour est fait sur la parole pour en tirer un apprentissage (Dumais, 2008). Il privilégie un travail spécifique sur l'oral puisqu'il permet d'observer, d'analyser, de manipuler, d'exercer et d'étudier les objets de l'oral et de réfléchir à leur utilisation. 


\subsection{Les interventions du dispositif de formation}

Le tableau 2 présente les interventions qui font partie du dispositif de formation en explicitant de façon plus précise ce qu'il contient et ce qu'il vise par rapport aux statuts de l'oral (oral objet ou oral médium).

Tableau 2

Le dispositif de formation et les interventions ayant l'oral comme objet ou comme médium

\begin{tabular}{|c|c|c|}
\hline TYPES & $\begin{array}{c}\text { Oral } \\
\text { comme } \\
\text { Objet }\end{array}$ & $\begin{array}{c}\text { Oral } \\
\text { comme } \\
\text { Médium }\end{array}$ \\
\hline \multicolumn{3}{|l|}{ Libre et facultatif (LF) } \\
\hline - Centre d'aide en français & $\mathrm{X}$ & \\
\hline - Site internet du test oral & $\mathrm{X}$ & \\
\hline \multicolumn{3}{|l|}{ Diagnostique et de remédiation (DR) } \\
\hline - Test de l'oral & $\mathrm{X}$ & \\
\hline $\begin{array}{l}\text { - Cours obligatoires à la suite de l'échec au test } \\
\text { de l'oral (LIN... ) }\end{array}$ & $\mathrm{X}$ & \\
\hline $\begin{array}{l}\text { Cours obligatoire en didactique de l'oral } \\
\text { (DDL2735) }\end{array}$ & $\mathrm{X}$ & \\
\hline \multicolumn{3}{|l|}{ Non interventionniste (NI) } \\
\hline - Stages & & $\mathrm{X}$ \\
\hline $\begin{array}{ll}\text { - Analyses réflexives dans les stages avec } \\
\text { enseignants associés et superviseurs }\end{array}$ & & $\mathrm{X}$ \\
\hline - Prestations orales dans les cours & & $\mathrm{X}$ \\
\hline \multicolumn{3}{|l|}{ Transversal et progressif (TP) } \\
\hline - Formation spécifique sur l'oral & $\mathrm{X}$ & \\
\hline $\begin{array}{llll}\text { - Quatre situations } & \text { d'apprentissage } & \text { et } \\
\text { d'évaluation (SAÉ) } & & \\
\end{array}$ & $\mathrm{X}$ & $\mathrm{X}$ \\
\hline - Grille d'autoévaluation et de coévaluation & $\mathrm{X}$ & \\
\hline
\end{tabular}

Les interventions de type libre et facultatif (LF) sont à la disposition des étudiants en tout temps. Pour en profiter, ils doivent consulter le site internet du test oral ou rencontrer les moniteurs du Centre d'aide en français selon leurs besoins. Les interventions de type diagnostique et de remédiation (DR) visent à encadrer les étudiants de manière plus formelle. Elles sont prévues dans le cheminement de l'étudiant et elles sont obligatoires ${ }^{7}$. Le test mesurant les habiletés orales des futurs enseignants se réalise au mois de mai de la première session. En cas d'échec au test, les étudiants doivent suivre un ou plusieurs cours hors programme pour améliorer

\footnotetext{
${ }^{7}$ Depuis l'automne 2013, le test oral n'est plus obligatoire, mais est cependant fortement recommandé.
} Les étudiants qui décident de ne pas faire le test sont dans l'obligation de suivre les cours d'appoint. 
leur compétence à l'oral. Ces cours ne sont pas à suivre pour ceux qui réussissent le test. Le cours de didactique de l'oral (DDL2735), qui permet aux futurs enseignants d'apprendre à enseigner et à évaluer l'oral, se donne en première année de formation. Dans ce cours, les étudiants prennent entre autres connaissance des différents objets de l'oral dans un contexte d'enseignement au primaire, ce qui leur permet par la même occasion de connaitre et de comprendre les objets à développer pour améliorer leur compétence à l'oral. Également, dans ce cours, en plus d'élaborer une situation d'apprentissage en communication orale pour une année scolaire donnée, les étudiants effectuent une autoévaluation de leur compétence à l'oral, soit un autoportrait, ce qui leur permet de faire des liens entre le développement de leur compétence à l'oral et les objets de l'oral qu'ils auront à enseigner à leurs élèves. Les interventions de type non interventionniste (NI) se déroulent dans les stages ou dans les cours et proposent des occasions particulières où les étudiants ont l'opportunité de mettre la grille d'autoévaluation et de coévaluation (Dumais et al., 2013) en application. Aucun enseignement explicite n'est effectué de la part de l'enseignant associé ou de l'enseignant du cours où la grille est utilisée. Seuls les échanges sont encouragés. Enfin, les interventions de type transversal et progressif (TP) permettent la pratique des objets de l'oral en tenant compte des années de formation et des situations d'apprentissage et d'évaluation (SAÉ) appropriées pour chacune d'entre elles. Un enseignement explicite est effectué pour comprendre les objets de l'oral.

\section{Méthodologie}

Afin de pouvoir identifier les interventions déclarées efficaces par les étudiants du programme ÉPEP de l'UQAM, nous avons d'abord procédé à une collecte de données par questionnaire. Par la suite, afin de pouvoir approfondir certains des résultats obtenus par l'enquête par questionnaire, nous avons tenu un groupe de discussion avec des étudiants de $3^{\mathrm{e}}$ année du baccalauréat ayant répondu au questionnaire.

En ce qui concerne le questionnaire, il avait comme objectif de répondre à notre question de recherche qui est d'identifier les interventions qui, selon les étudiants consultés, sont perçues comme les plus favorables au développement des compétences à l'oral des étudiants du programme. La population touchée par le questionnaire était tous les étudiants inscrits au baccalauréat en éducation préscolaire et en enseignement primaire à l'automne 2013, moment prévu pour la passation du questionnaire. Ainsi, nous touchions les étudiants du programme qui avaient pu vivre certaines des initiatives qui ont été mises en place dans le cadre de leur formation depuis 2010 et ceux qui débutaient leur formation initiale. Afin de constituer notre échantillon, de nature non probabiliste, les étudiants de la $2^{\mathrm{e}}$, de la $3^{\mathrm{e}}$ et de la $4^{\mathrm{e}}$ année qui avaient vécu plusieurs des interventions présentées précédemment (voir tableau 2) ont été approchés pour participer à l'enquête par questionnaire. Les étudiants de $1^{\text {re }}$ année ont aussi été approchés afin de recueillir les perceptions qu'ils entretenaient sur l'utilité des interventions qu'ils pourraient avoir eues lors de formations antérieures, au collégial notamment. Le questionnaire a alors été adapté pour correspondre à la réalité de ces étudiants commençant leur formation initiale. Le questionnaire a donc été proposé à l'ensemble de ces étudiants, ce qui nous a conduits à interroger 563 
étudiants au total (annexe 1). Dans la figure suivante, la répartition des étudiants par année de baccalauréat qui ont répondu au questionnaire est présentée.

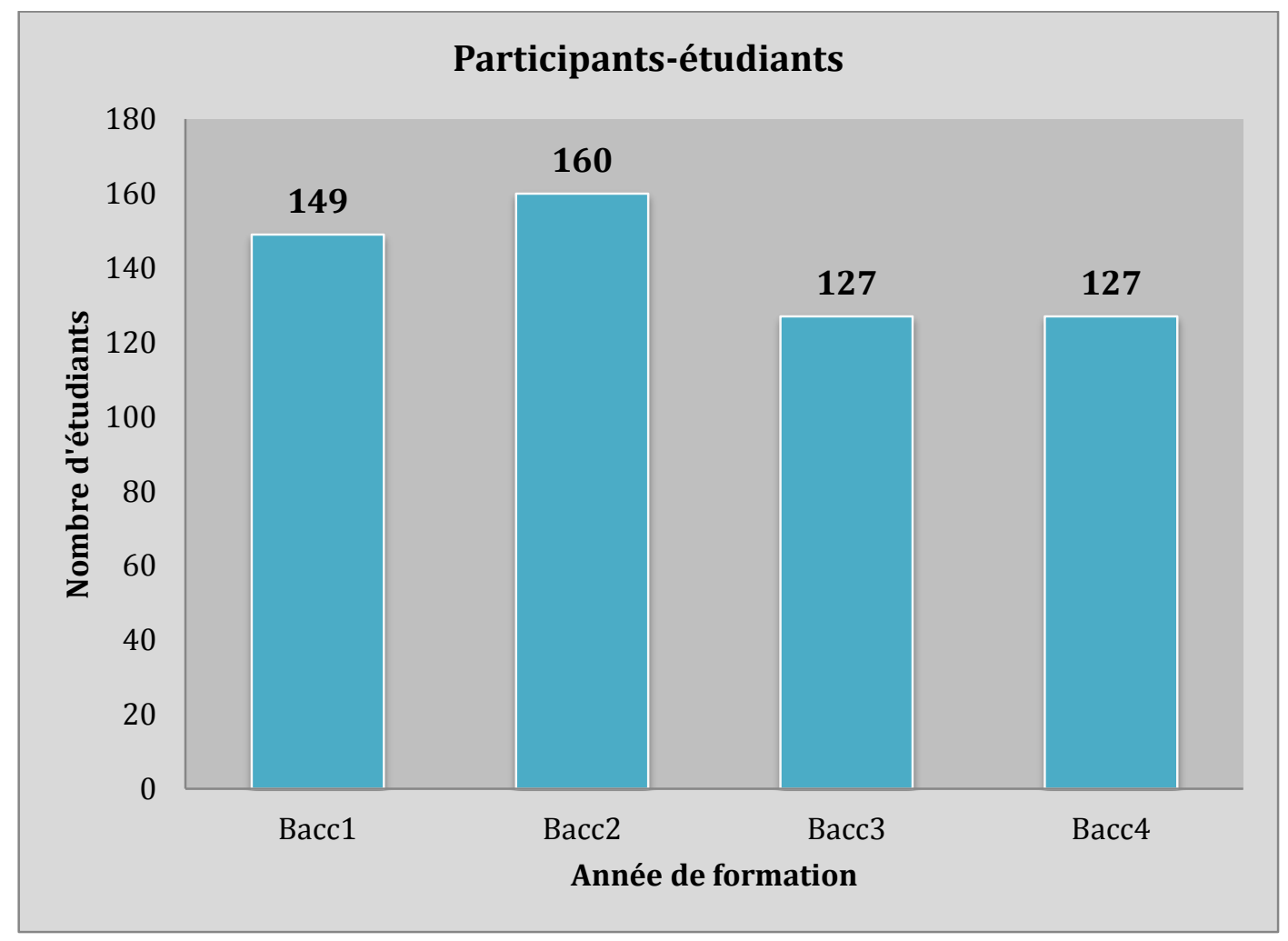

Figure 1. Répartition des étudiants qui ont participé à l'enquête par questionnaire par année de baccalauréat $(\mathrm{n}=563)$

Dans le questionnaire, les étudiants ont été invités à nous transmettre leurs coordonnées s'ils souhaitaient participer à un entretien de groupe permettant d'approfondir certains résultats de l'enquête. Nous avons donc sollicité dans un premier temps les étudiants de la $3^{\mathrm{e}}$ année du baccalauréat qui avaient consenti à participer à ces entretiens ${ }^{8}$. Nous avons réussi à en réunir cinq. Nous avons privilégié un entretien semi-structuré, laissant ainsi la place à des questions ouvertes en lien avec les résultats obtenus avec le questionnaire, mais aussi à des questions imprévues. Nous avons privilégié l'analyse de contenu comme technique pour analyser les entretiens semi-structurés (Bardin, 2003; L’Écuyer, 1987, 1990), en privilégiant un codage ouvert, c'est-à-dire un codage où « le lexique se composera en cours de codage » (Van der Maren, 1996, p. 136).

La prochaine section présente les résultats croisés de l'enquête avec ce qui ressort de l'entretien mené avec les étudiants de la $3^{\mathrm{e}}$ année du baccalauréat.

\footnotetext{
${ }^{8}$ Comme les stages pour les cohortes de $1^{\text {re }}, 2^{\mathrm{e}}$ et $4^{\mathrm{e}}$ années se déroulent pendant la session d'hiver, il
} était plus aisé de commencer les entretiens avec les étudiants de $3^{\mathrm{e}}$ année. 


\section{Résultats}

Nous avons choisi d'organiser la présentation des résultats dans cette publication selon le type d'intervention privilégié. Des résultats en lien avec les interventions de type diagnostique et de médiation, ceux de type non interventionniste et ceux de type transversal et progressif seront donc donnés, en mettant spécifiquement l'accent sur les interventions mentionnées à la fois dans les résultats de l'enquête et de l'entretien de groupe. Comme les interventions de type libre et facultatif n'ont pas fait l'objet de discussions lors de l'entretien de groupe analysé, nous avons choisi de ne pas mettre l'accent sur ces résultats dans le cadre de cette publication.

\subsection{Les interventions de type diagnostique et de médiation}

Lors de l'analyse des résultats en lien avec ces interventions, deux des interventions ont retenu l'attention : le cours de didactique de l'oral que suivent les étudiants en première année du baccalauréat et les cours obligatoires que doivent faire les étudiants à la suite d'un échec au test oral. Rappelons que le test oral a lieu à la fin de la première année de la formation ÉPEP et que les cours à suivre à la suite d'un échec peuvent être suivis par choix si les étudiants choisissent de ne pas effectuer le test oral.

À propos du cours de didactique de l'oral dispensé en formation initiale, dont l'objectif principal n'est pas de développer les compétences à l'oral des étudiants, mais plutôt de favoriser l'acquisition des fondements d'une didactique de l'oral pour leur pratique enseignante, les étudiants affirment, dans l'ensemble, que ce cours a une incidence favorable sur le développement de leur compétence à l'oral. En effet, les étudiants de $2^{\mathrm{e}}$ et de $3^{\mathrm{e}}$ année le considèrent comme une intervention de formation favorable à 90/160 et 74/127, alors que 58/127 des étudiants de $4^{\text {e }}$ année abondent dans le même sens (figure 2). Lors de l'entretien de groupe avec les étudiants de $3^{\mathrm{e}}$ année, ces derniers ont précisé que le cours de didactique de l'oral leur a entre autres donné l'occasion de prendre conscience qu'ils ne sont pas d'emblée compétents à l'oral, ce qu'ils tenaient pour acquis lors de leur entrée en formation initiale, en plus de leur permettre d'identifier leurs forces et leurs faiblesses en contexte formel par la réalisation d'un portrait du locuteur (Dumais, 2015). 


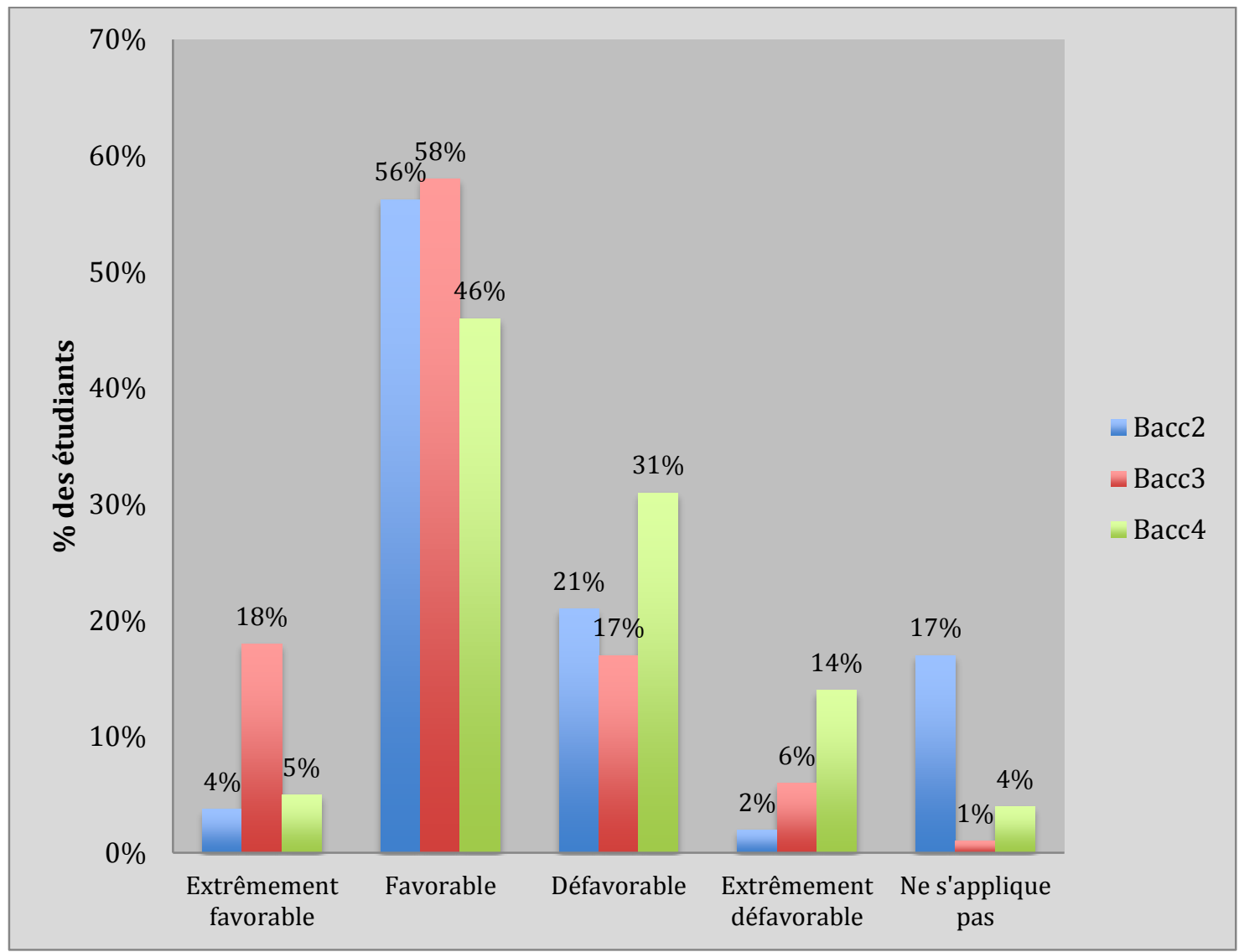

Figure 2. Résultats de l'enquête à propos des perceptions des étudiants sur l'efficacité du cours de didactique de l'oral (DDL 2735)

Certains étudiants suivent des cours de communication orale qui ne sont pas intégrés dans le cursus des cours du programme de formation. Ces cours sont obligatoires pour les étudiants qui échouent au test oral prévu à la fin de la première année de baccalauréat ou pour ceux qui choisissent de ne pas passer le test oral, qui est facultatif. Parmi les répondants qui ont eu à les faire (environ $68 \%$ des étudiants qui ont répondu au questionnaire), plusieurs soulignent que ces cours constituent une intervention de formation favorable dans le développement de leur compétence en communication orale. On peut remarquer qu'ils l'ont été davantage pour les étudiants finissants que nous avons interrogés $(n=52)$. 


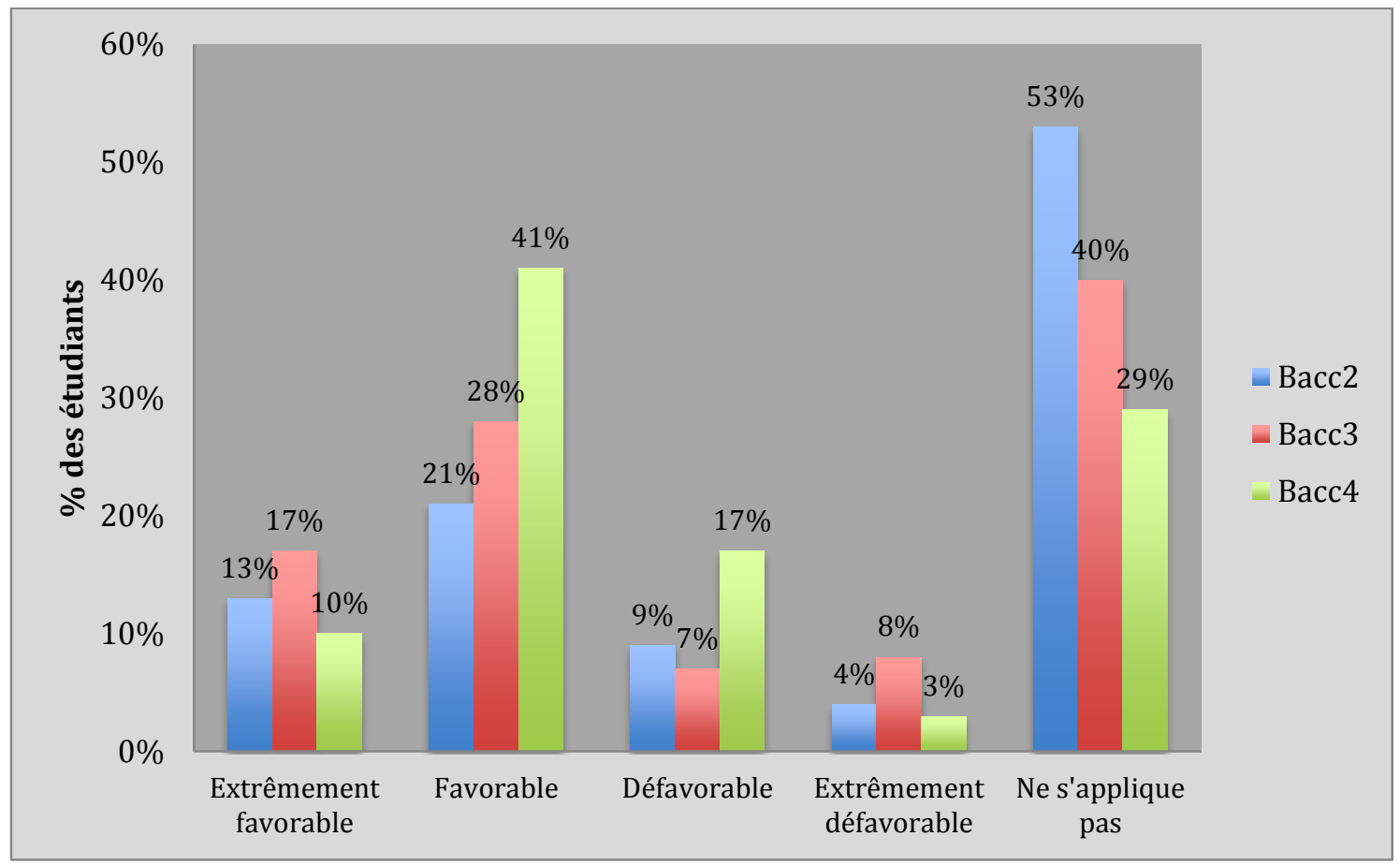

Figure 3. Résultats de l'enquête à propos des perceptions des étudiants sur l'efficacité des cours obligatoires à la suite d'un échec au test oral ou suivis par choix

Lors de l'entretien avec les étudiants de $3^{\mathrm{e}}$ année, certains des sujets ayant participé à ces cours ont souligné qu'ils permettaient l'apprentissage d'objets d'enseignement/apprentissage liés à l'oral, mais que comme leur apprentissage était réalisé dans des situations de communication non authentiques (par exemple, des ateliers de travail en ilots sur des objets ciblés), ils considéraient ces apprentissages comme difficilement transférables en situation de communication authentique. Aussi, les étudiants ont rapporté avoir vécu une évaluation d'une prestation orale qu'ils ont trouvée peu naturelle et très stressante. Ils proposaient alors d'être évalués dans une situation de communication plus authentique, tel un groupe de discussion, évaluation qui inclurait une autoévaluation de leur prestation afin de pouvoir en apprendre davantage sur leur compétence à l'oral. La grande majorité des étudiants du groupe de discussion ont précisé que ces cours devraient être inclus obligatoirement dans le curriculum de formation, en faisant le parallèle avec les cours qu'ils suivent en lien avec leur compétence à l'écrit, afin de mettre en évidence l'importance de la compétence à l'oral dans un contexte professionnel.

\subsection{Les interventions de type non interventionniste}

Lorsqu'il est question des interventions de type non interventionniste, l'analyse révèle des résultats inattendus en lien avec les stages effectués dans le cadre de la formation initiale et les prestations orales réalisées dans les cours.

En effet, selon l'ensemble des étudiants qui ont participé à l'enquête, près de $62,8 \%$ d'entre eux considèrent les stages, de façon générale, comme étant une intervention de formation très favorable au développement de leur compétence à 
l'oral, et $24 \%$ comme favorable (figure 4). Nous pouvons donc affirmer que les stages supervisés s'avèrent une occasion et un lieu propices au développement de leur compétence à l'oral, et non une intervention en soit, ce à quoi les étudiants rencontrés lors de l'entretien de groupe ont acquiescé. Ils nous ont précisé que les stages les plaçaient dans un contexte naturel et authentique, ce qui les forçait à accorder plus d'attention à leur compétence à l'oral. Toujours selon eux, les stages sont une occasion de prendre conscience de leurs forces et faibles, et de l'impact de leur communication orale sur les apprentissages des élèves. Certains ont souligné que les rétroactions reçues pendant le stage, les captations vidéo de leur prise en charge ou les rencontres avec les superviseurs pendant les stages favorisaient cette prise de conscience.

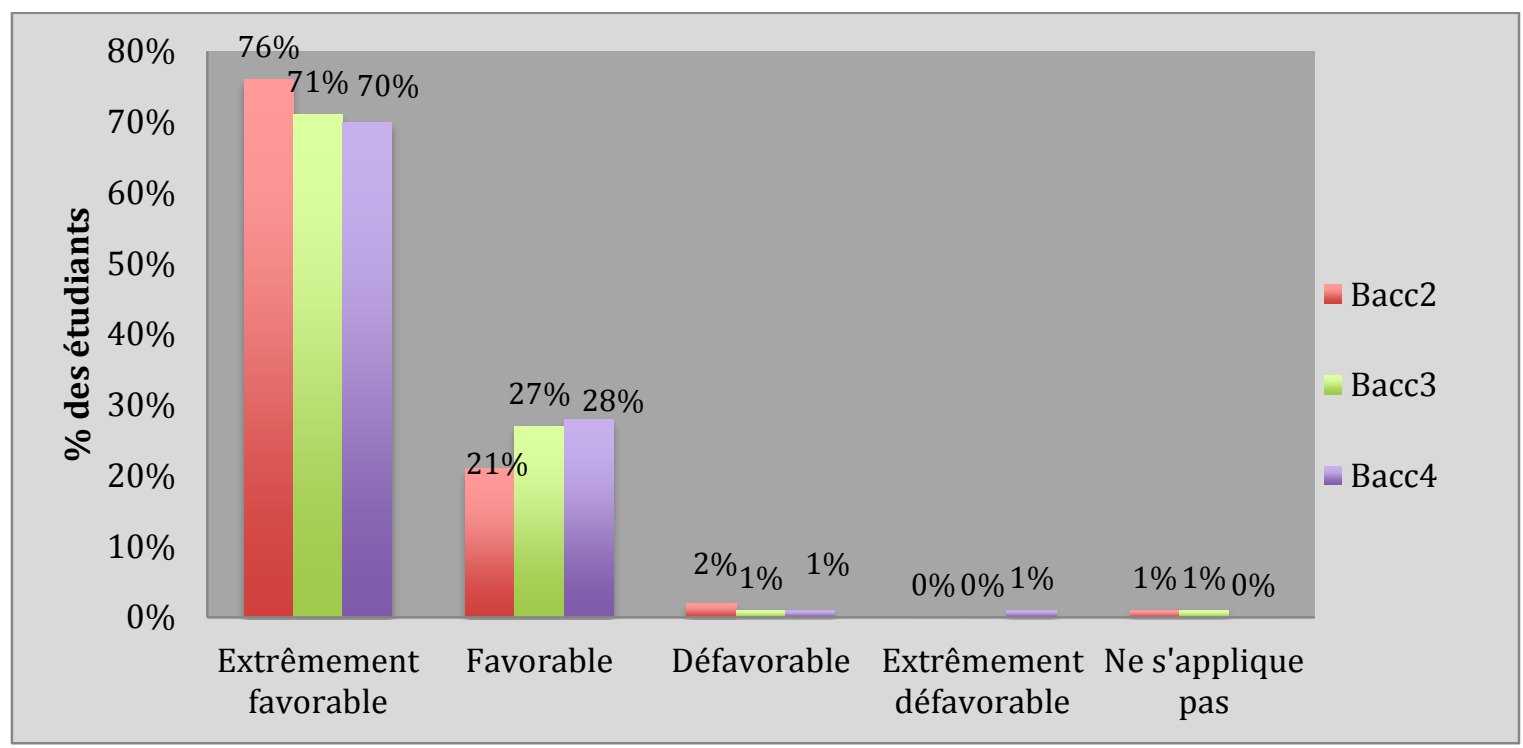

Figure 4. Résultats de l'enquête à propos des perceptions des étudiants sur l'efficacité des stages

Nous avons aussi questionné les étudiants afin de savoir s'ils estimaient que les entretiens avec les enseignants associés étaient un moment privilégié pour développer leur compétence à l'oral. Selon les résultats obtenus avec le questionnaire, environ $46 \%$ des étudiants consultés soulignent que ces entretiens sont très favorables alors que $38 \%$ d'entre eux les considèrent comme favorables (figure 5). Lors des entretiens, les étudiants ont en effet réitéré la pertinente des entretiens avec les enseignants associés pour identifier des erreurs récurrentes qu'ils font à l'oral (par exemple, la présence de tics ou la répétition de mots sont soulignées comme des erreurs mentionnées par les enseignants associés). Toutefois, certains se questionnaient sur la compétence en communication orale des enseignants associés («sont-ils plus compétents que nous sur le plan de la communication orale?»), mais aussi de l'accompagnement possible qu'ils peuvent leur fournir pour qu'ils développent leur compétence à l'oral («sont-ils véritablement en mesure de nous aider à être plus compétents? »). 


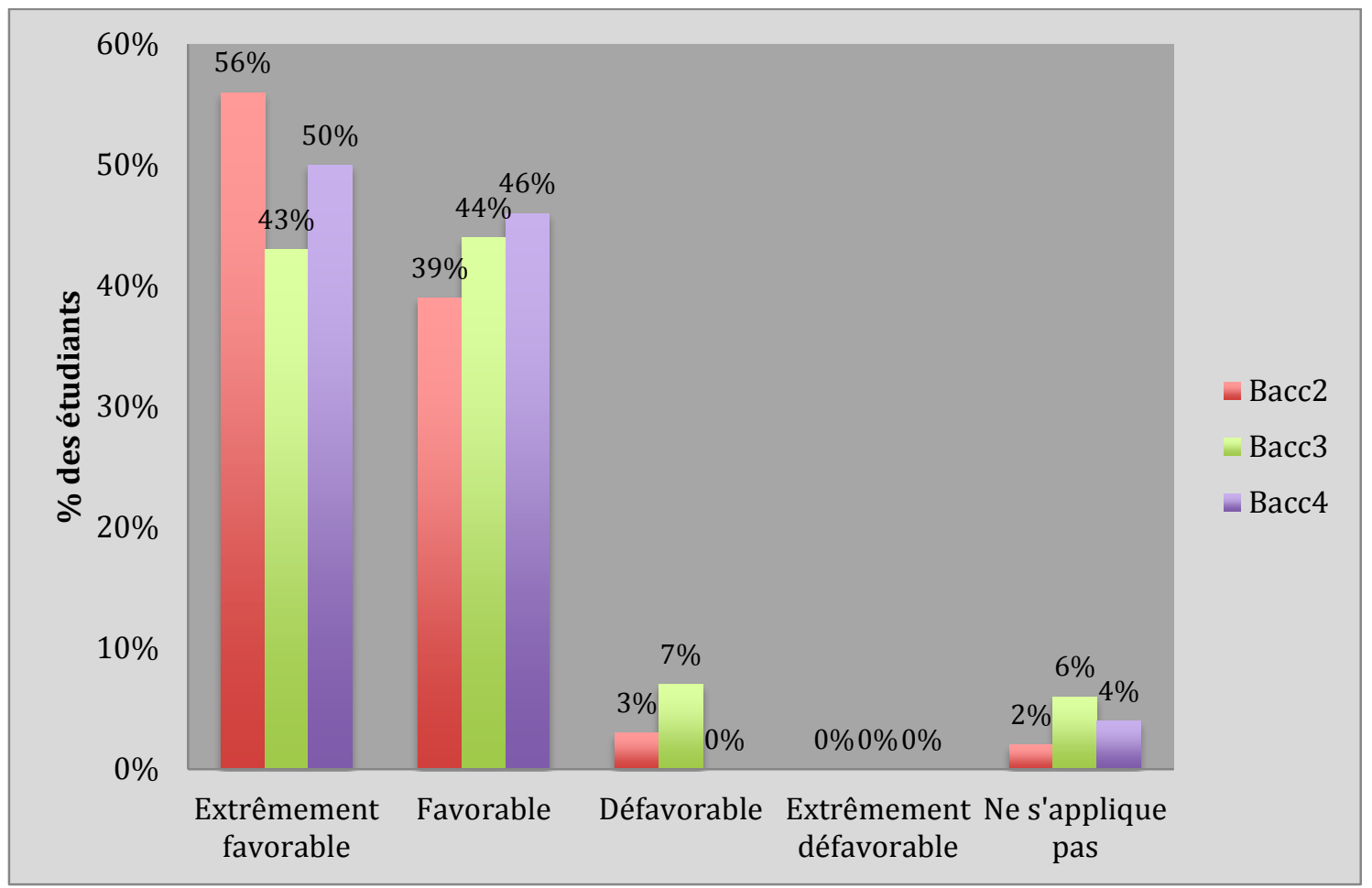

Figure 5. Résultats de l'enquête à propos des perceptions des étudiants sur l'efficacité des entretiens avec les enseignants associés pendant les stages

En ce qui a trait plus spécifiquement aux rencontres avec les superviseurs encadrant les stages ayant lieu en cours de formation, la majorité des étudiants ont souligné que celles-ci étaient extrêmement favorables (45\%) ou favorables $(42 \%)$ pour soutenir le développement de leur compétence à l'oral (figure 6). Lors de l'entretien de groupe, les étudiants rencontrés ont précisé, entre autres, qu'ils étaient soucieux de mieux performer à l'oral lors des supervisions afin que les superviseurs puissent les observer et leur donner de la rétroaction ou leur proposer des exercices de remédiation en conséquence. 


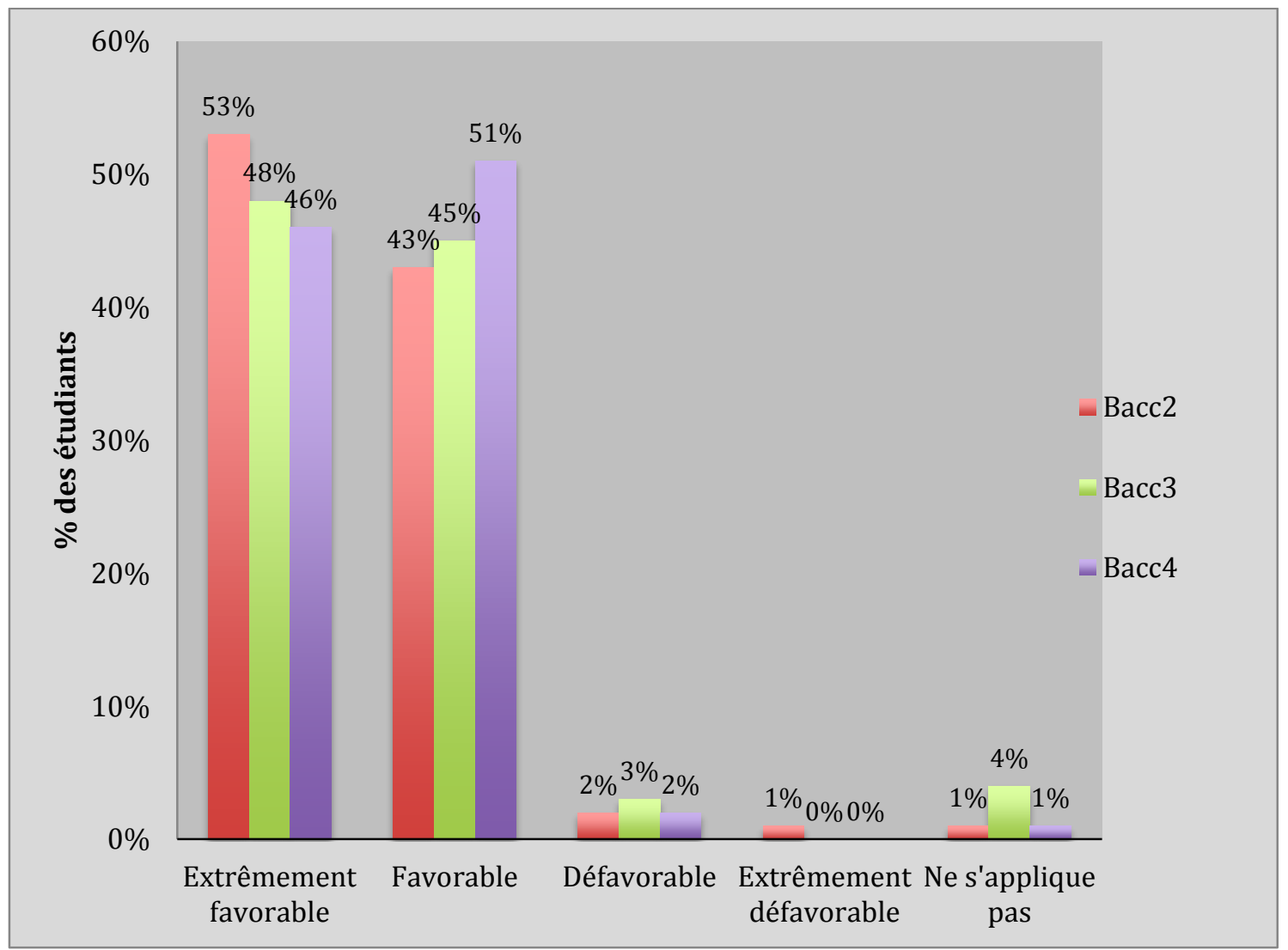

Figure 6. Résultats de l'enquête à propos des perceptions des étudiants sur l'efficacité des entretiens avec les superviseurs de stage

Les prestations orales effectuées dans les cours en formation initiale ont été considérées comme favorables par 66,8 \% des étudiants consultés (figure 7). Lorsque nous avons interrogé les étudiants en entretien de groupe sur cette intervention de formation, ils ont confirmé la pertinence de ces prestations même si, bien souvent, leur évaluation porte exclusivement sur la compétence discursive, au détriment des autres volets de l'oral. Ils ont souligné le fait que ces prestations devaient être évaluées sur le plan de la communication orale de façon globale et non seulement sur le contenu des prestations, et qu'une formation plus solide en ce qui a trait au développement de cette compétence serait un atout considérable dans le cadre de la formation initiale. 


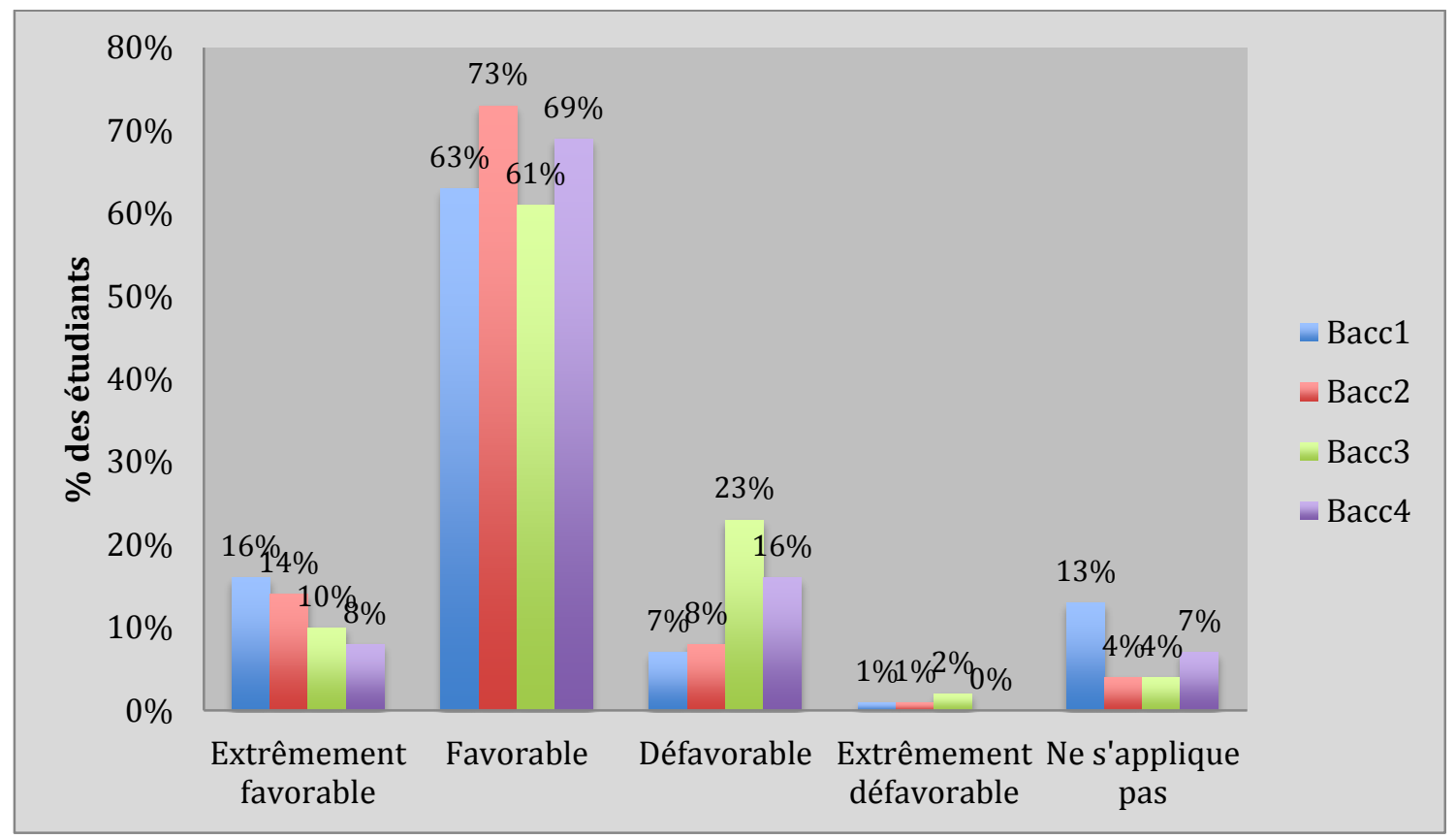

Figure 7. Résultats de l'enquête à propos des perceptions des étudiants sur l'efficacité des prestations orales effectuées dans les cours en formation initiale

La prochaine section aborde les résultats obtenus en lien avec les interventions de type transversal et progressif.

\subsection{Les interventions de type transversal et progressif}

Dans les interventions de type transversal et progressif (TP), c'est la grille de coévaluation et d'autoévaluation qui est rapportée comme la plus favorable au développement de la compétence à l'oral auprès des étudiants de $2^{\mathrm{e}}$, de $3^{\mathrm{e}}$ et de $4^{\mathrm{e}}$ année interrogés, soit à $49 \%$ (figure 8). Lors de l'entretien de groupe, afin que la grille soit exploitée davantage en formation initiale, les étudiants ont proposé qu'elle soit disponible dans un format plus convivial (proposition d'un format de poche) afin qu'elle soit employée tant en contexte formel qu'en contexte informel dans le cadre de la formation. Aussi, les étudiants ont proposé que la grille soit employée pour l'analyse des captations vidéo en stage. 


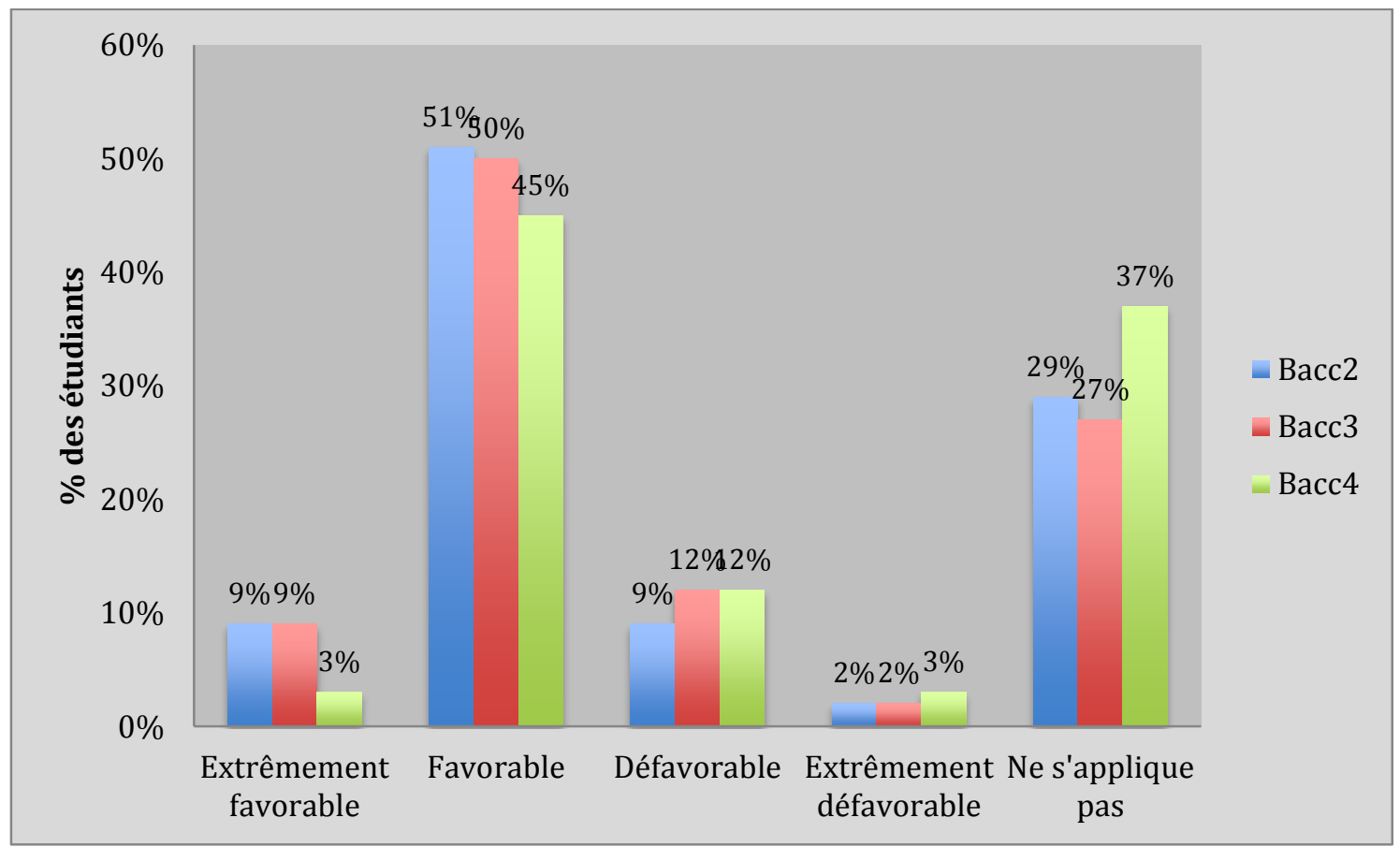

Figure 8. Résultats de l'enquête à propos des perceptions des étudiants sur l'efficacité de la grille d'autoévaluation et de coévaluation

La prochaine section présente une discussion des résultats que nous venons de présenter.

\section{Discussion}

Tout comme pour la section précédente, la discussion des résultats sera organisée par type d'encadrement, soit selon les interventions de type diagnostique et de médiation, sur les interventions de type non interventionniste et sur les interventions de type transversal et progressif qui composent le dispositif de formatif proposé aux étudiants du programme ÉPEP.

\subsection{Discussion sur les interventions de type diagnostique et de remédiation}

Il est possible de constater que les interventions de type diagnostique et de remédiation telles que le cours de didactique de l'oral ou les cours obligatoires que doivent faire les étudiants à la suite d'un échec (ou non) au test oral placent les étudiants devant des faits observables et mesurables sur l'état actuel de leur compétence à l'oral, ce qu'ils n'ont pas eu souvent l'occasion de faire dans leur parcours scolaire (Fisher, 2007). Les résultats que nous avons présentés au sujet des interventions de type diagnostique et de médiation mises en place au programme ÉPEP de l'UQAM nous amènent à croire qu'il est possible de développer une réflexion métalinguistique chez les étudiants en formation initiale, en plus des savoirs propres à la communication orale ou à la didactique de l'oral. Même si certains écrits existants sur la question soulignent que les étudiants en formation initiale sont peu conscients de leurs forces et de leurs faiblesses (Ostiguy, 2005 ; Lebrun, 2008), nos résultats abondent dans le sens de la recherche de Tremblay, Mottet et Chevrette 
(2014). Selon ces chercheuses (2014, p. 12), « les étudiants sont plutôt attentifs à leur façon de s'exprimer et cherchent à "améliorer" leur expression orale ».

Les interventions de type diagnostique et de remédiation sont considérées comme pertinentes par les étudiants au point de vouloir qu'elles soient obligatoires dans la formation. Cela rejoint les propos de Fisher (2007) selon lesquels il faut intervenir de façon explicite en formation initiale afin que les futurs enseignants considèrent qu'il est possible d'améliorer leur capacité à communiquer oralement par un enseignement de l'oral. Ce constat rejoint aussi de nombreux travaux qui ont démontré que l'oral nécessite d'être enseigné et qu'il est possible de réellement l'enseigner (Dolz et Schneuwly, 1998; Le Cunff et Jourdain, 1999; Lafontaine, 2001; Messier, 2004; Dumais, 2008). De plus, ces interventions doivent donner l'occasion aux étudiants de prendre conscience de la qualité de leur langue orale puisqu'ils seront des modèles linguistiques et que le degré de maitrise de cette compétence aura des répercussions directes dans leur enseignement (Lebrun, 2008 ; Mottet, 2009; Daviault, 2011; Dumais et al., 2013; Dumais, 2015).

Malgré la pertinence de ces interventions en ce qui a trait au développement de la compétence à l'oral, soulignons qu'elles n'offrent pas toujours des contextes de prises de parole authentiques comme celles des stages, ce qui pourrait être favorisé davantage en formation initiale.

\subsection{Discussion sur les interventions de type non interventionniste}

Les résultats de l'enquête réalisés confirment le constat de Fisher (2007) sur le fait que les compétences à l'oral ne peuvent pas se développer en dehors d'un contexte professionnel dans lequel elles sont requises, le stage étant le lieu par excellence dans ce cas-ci. Ces résultats concordent assez bien avec des situations similaires en formation initiale des enseignants voulant que les étudiants croient que la plus grande partie de leurs apprentissages se font dans les stages (Viola, 2011). Malgré la place importante accordée aux stages par les étudiants qui ont répondu à l'enquête et qui ont participé à l'entretien de groupe, le développement réel des compétences à l'oral dans un contexte de stage serait à questionner comme les enseignants associés et les superviseurs de stage semblent peu formés à propos de l'oral et des objets qui le concernent, ce qu'ont d'ailleurs relevé certains étudiants lors des entretiens. Les travaux de Lafontaine (2005) faisaient déjà état de cette situation. Cependant, comme le précisent Chaubet et Gervais (2014), les stages supervisés constituent les situations les plus porteuses pour la transformation des schèmes de pensée et d'action. 


\subsection{Discussion sur les interventions de type transversal et progressif}

Comme il a été possible de le constater, les interventions de type transversal et progressif sont considérées comme favorables pour soutenir le développement de la compétence à l'oral des étudiants. Nos résultats concordent avec les recherches portant sur les caractéristiques des étudiants des années 2000 : ils sont autonomes, engagés et pragmatiques (Garceau, 2012). Ils veulent des formations qui répondent à leurs besoins ici et maintenant. Ils sont aussi sensibles aux interventions individualisées et souhaitent un suivi de la part des enseignants. La formation et le développement personnel sont prioritaires pour eux (Prégent, Bernard et Kozanitis, 2009). Les propos des étudiants vont dans le même sens que Tremblay (2003) qui affirme que pour que des interventions soient efficaces et utiles aux futurs enseignants, elles doivent être mises en pratique régulièrement et doivent leur paraitre bénéfiques afin qu'ils prennent l'habitude de les utiliser, ce qu'il est possible d'envisager avec la grille d'autoévaluation et de coévaluation élaborée dans le cadre de nos travaux amorcés en 2010 (Dumais et al., 2013).

\section{Conclusion}

Afin de soutenir le développement de la compétence à l'oral des futurs enseignants inscrits dans le programme ÉPEP de l'UQAM, qui préconise l'approcheprogramme, plusieurs interventions ont été créées et mises en place depuis 2010. Après quelques années d'expérimentation, nous avons donc cherché à connaitre les interventions les plus favorables au développement des compétences à l'oral telles que perçues par les étudiants du programme. Un questionnaire a donc été passé aux étudiants de la $1^{\text {re }}$ à la $4^{\mathrm{e}}$ année inscrits à la session d'automne 2013. À la suite de cette enquête, un entretien de groupe a été réalisé avec cinq étudiants de la $3^{\mathrm{e}}$ année. Les résultats nous ont permis d'identifier les interventions de type non interventionniste, nommément les stages, comme étant perçues les plus favorables pour le développement de la compétence à l'oral des étudiants, ces derniers se réalisant en situation de communication authentique. Les étudiants expliquent alors être beaucoup plus conscients de l'importance de bien communiquer oralement et font alors un effort supplémentaire pour mieux communiquer. Bien que les interventions de type diagnostique et de médiation, tels les cours déjà présents dans le programme actuel, soient considérées comme ayant une incidence favorable sur le développement de la compétence à l'oral, une formation plus complète dont l'objectif principal serait de développer tous les volets de la communication orale est jugée nécessaire pour que les étudiants se sentent plus compétents. Les résultats mettent aussi de l'avant le bénéfice des interventions telles que la grille d'autoévaluation et de coévaluation où les étudiants ont à s'autoévaluer ou sont évalués comme des occasions supplémentaires d'apprentissage. 


\section{Références}

Bardin, L. (2003). L'analyse de contenu (11 ${ }^{\mathrm{e}}$ éd.). Paris : Presses universitaires de France.

Basque, J. et Rogozan, D. (2013). Vers une méthode d'ingénierie de l'approcheprogramme en enseignement supérieur. Dans Bédard, D. (dir.), Les innovations pédagogiques en enseignement supérieur: Pédagogies actives en présentiel et à distance, Actes du VIIe colloque Questions de pédagogies dans l'enseignement supérieur (p. 578-588). Sherbrooke : Université de Sherbrooke.

Caron, P.-A. (2007). Contextualisation de dispositifs pédagogiques sur des applications Web 2.0, Actes du colloque : Actualité de la Recherche et de l'Éducation en Formation (AREF), Strasbourg. Récupéré le 20 janvier 2015 de http://www.congresintaref.org/acte_cd.php?act=show\&cont_id=501

Chaubet, P. et Gervais, C. (2014). Analyser l'alternance d'enquêtes entre pratique et théorie pour mieux les provoquer ? Éducation et francophonie, 42 (1), 149-166

Daviault, D. (2011). L'émergence et le développement du langage chez l'enfant. Montréal : Chenelière éducation.

Desjardins, J. et Dezutter, O. (2009). Développer des compétences professionnelles en formation initiale à l'enseignement préscolaire et primaire : regard sur l'organisation des programmes en contexte québécois, Canadian Journal of Education, 32(4), 873-902.

Dolz, J. et Schneuwly, B. (1998). Pour un enseignement de l'oral: Initiation aux genres formels à l'école. Paris: ESF éditeur.

Dumais, C. (2008). Effets de l'évaluation par les pairs sur les pratiques d'expression orale d'élèves de troisième secondaire: une description. Mémoire de maîtrise inédit. Montréal : Université du Québec à Montréal.

Dumais, C. (2015). Une typologie des objets de l'oral pour la formation initiale et continue des enseignants. Dans R. Bergeron, C., Dumais, B., Harvey et R. Nolin (Dir.). Didactique du français oral du primaire à l'université (p. 29-52). Côte Saint-Luc : Éditions Peisaj.

Dumais, C. et Goyette, N. (2012). Évaluation de la compétence à communiquer à l'écrit et à l'oral en formation initiale des maîtres : état de la situation au Québec. Actes du 24e Colloque international de l'Association pour le développement des méthodologies d'évaluation en éducation - Europe (ADMEE- Europe): L'évaluation des compétences en milieu scolaire et en milieu professionnel. Luxembourg ville, Luxembourg. Récupéré le 19 janvier 2015 de http://admee2012.uni.lu/pdf2012/A08_02.pdf

Dumais, C., Messier, G., Viola, S. et Meunier, H. (2013). Des grilles d'autoévaluation et de coévaluation pour améliorer la compétence orale. Québec français, 170, 64-68.

Fisher, C. (2007). Le développement des compétences langagières à l'oral dans le contexte de la formation à l'enseignement. Dans É. Falardeau, C. Fisher, C. Simard et N. Sorin (Dir.), La didactique du français : Les voies actuelles de la recherche (p. 257-274). Québec : Presses de l'Université Laval

Garceau, J. (2012). La cohabitation des générations. Montréal : Les éditions La presse. 
Gouvernement du Québec (2001a). La formation à l'enseignement. Les orientations. Les compétences professionnelles. Québec : Ministère de l'Éducation du Québec.

Gouvernement du Québec (2001b). Le français, une langue pour tout le monde - Une nouvelle approche stratégique et citoyenne. Québec: Commissions des états généraux sur la situation et l'avenir de la langue française au Québec. Récupéré le 19 janvier 2015 de http://www.spl.gouv.qc.ca/documentation/rapportssondages statistiques/

Lafontaine, L. (2001). Élaboration d'un modèle didactique de la production orale en classe de français langue maternelle au secondaire. Thèse de doctorat inédite. Montréal : Université du Québec à Montréal

Lafontaine, L. (2005). La place de la didactique de l'oral en formation initiale des enseignants de français langue d'enseignement au secondaire. Nouveaux cahiers de la recherche en éducation, 8, 1, 95-109.

Lebrun, M. (2008). La compétence à communiquer oralement et la compétence linguistique chez les enseignants en formation. Vie pédagogique, 149, 53-56.

Le Cunff, C. et Jourdain, P. (1999). Enseigner l'oral à l'école primaire. Paris : Hachette Éducation.

L'Écuyer, R. (1987). L'analyse de contenu : Notion et étapes. Dans J.-P. Deslauriers (Dir.), La recherche qualitative : Résurgence et convergences (p. 49-66). Ste-Foy, Québec : Presses de l’Université du Québec.

L'Écuyer, R. (1990). Méthodologie de l'analyse développementale de contenu méthode GPS et concept de soi. Sillery : Presses de l'Université du Québec.

Messier, G. (2004). Enseignement/apprentissage de l'oral en classe de français langue maternelle au secondaire: mise en place d'un modèle didactique. Mémoire de maitrise inédit. Montréal : Université du Québec à Montréal.

Messier, G., Dumais, C. et Viola, S. (2012). Portrait des perceptions de la communication orale des étudiants de première année en formation des maîtres. Dans R. Bergeron et G. Plessis-Bélair (Dir.), Représentations, analyses et descriptions du français oral, de son utilisation et de son enseignement au primaire, au secondaire et à l'université (p. 97-112). Côte Saint-Luc : Éditions Peisaj.

Mottet, M. (2009). Parler en bon français : quelle représentation les futurs enseignants du français en ont-ils? Québec français, 155, p. 89-91.

Ostiguy, L. (2005). La maîtrise du français parlé dans l'enseignement et les médias. Dans A. Stefanescu et P. Georgeault (Dir.). Le français au Québec. Nouveaux défis (p. 471-487). Montréal : Conseil supérieur de la langue française.

Paquelin, D. et Choplin, H. (2003). Se former dans un dispositif ouvert et à distance : l'enjeu des régulations. Dans B. Albero (Dir.), Autoformation et enseignement supérieur : au-delà de l'effet de mode une conception éducative (p. 166-183). Paris : Hermès éditeur.

Paquette, G. (2002). L'ingénierie pédagogique : Pour construire l'apprentissage en réseaux. Sainte-Foy : Presses de l'Université du Québec.

Peeters, H. et Charlier, P. (1999). Contributions à une théorie du dispositif. Hermès, $25,15-23$. 
Plessis-Bélair, G. (2006). La didactique de l'oral dans le programme actuel de formation : Au cœur de l'enseignement et de l'apprentissage. Dans J. Loiselle, L. Lafortune et N. Rousseau (dir.), L'innovation en formation à l'enseignement: Pistes de réflexion et d'action (p. 105-122). Québec : Les Presses de l'Université du Québec.

Préfontaine, C., Lebrun, M. et Nachbauer, M. (1998). Pour une expression orale de qualité. Montréal : Les Éditions Logiques.

Prégent, R., Bernard, H. et Kosanitis, A. (2009). Enseigner à l'université dans une approche-programme. Un défi à relever. Montréal, Québec : Presses internationales polytechniques.

Tremblay, M. B. (2003). La communication chez les enseignants, savoir-être et savoir-faire pédagogiques. Montréal : Guérin universitaire.

Tremblay, O. et Mottet M. (2012). Représentations du français oral standard et de la langue d'enseignement chez de futurs maitres au primaire. Dans R. Bergeron et G. Plessis-Bélair. (dir.), Représentations, analyses et descriptions du français oral, de son utilisation et de son enseignement au primaire, au secondaire et à l'université (p. 81-96). Côte Saint-Luc : Éditions Peisaj.

Tremblay, O., Mottet, M. et Chevrette, V. (2014). Représentations et usages du français québécois oral standard: portrait de trois futures enseignantes au primaire. Lidil, Revue de linguistique et de didactique des langues, 50, 169-187.

Van der Maren, J. (1996). Méthodes de recherche pour l'éducation (2e éd.). Montréal : Presses de l'Université de Montréal.

Viola, S. (2011). Document de présentation du programme d'éducation préscolaire et d'enseignement primaire : caractéristiques du programme, tableaux-synthèse et plans de cours. Document inédit. Montréal : Université du Québec à Montréal.

Viola, S., Dumais, C. et Messier, G. (2012). Approche-programme et activités de communication orale en formation des maîtres selon un processus de répartition spiralaire des contenus. Dans R. Bergeron et G. Plessis-Bélair (Dir.). Représentations, analyses et descriptions du français oral, de son utilisation et de son enseignement au primaire, au secondaire et à l'université (p. 147-164). Côte Saint-Luc : Éditions Peisaj.

Viola, S., Messier, G., Dumais, C. et Meunier, H. (2015). Les familles de situations au service du développement de la compétence à l'oral des futurs enseignants. Dans R. Bergeron, C., Dumais, B., Harvey et R. Nolin (Dir.). Didactique du français oral du primaire à l'université (p. 187-209). Côte Saint-Luc : Éditions Peisaj.

Weisser, M. (2010). Dispositif didactique? Dispositif pédagogique? Situations d'apprentissage!, Questions Vives, 4(13), 291-303. Récupéré le 19 janvier 2015 de http://questionsvives.revues.org/271 
Biographies des auteurs

Sylvie Viola est professeure au département de didactique et directrice du bureau de formation pratique de l'Université du Québec à Montréal (UQAM). Dans le cadre de ses cours, elle enseigne les modèles d'apprentissage et d'enseignement ainsi que le portfolio de développement professionnel et culturel. De plus, elle forme les enseignants associés et les superviseurs de stage au questionnement, à l'approche métacognitive et à l'analyse réflexive. Dans le cadre de ses recherches, elle s'intéresse à la didactique professionnelle, aux dispositifs facilitant le transfert des savoirs dans la pratique ainsi qu'au développement des compétences à l'oral des étudiants en formation des maitres dans le contexte de l'approche-programme.

Geneviève Messier est professeure de didactique générale et formation pratique à l'Université du Québec à Montréal (UQAM). Elle a fait un stage postdoctoral sur les pratiques pédagogiques en littératie universitaire des formateurs universitaires dans le cadre de la formation initiale des enseignants. Dans le cadre de ses études doctorales, elle a proposé un réseau conceptuel qui précise et illustre la nature, la structure ainsi que la dynamique des concepts apparentés au terme méthode en pédagogie. Elle détient aussi une maitrise en linguistique, profil didactique des langues, dont l'objet de recherche se situe en didactique de l'oral. Elle a aussi enseigné au primaire et au secondaire en français langue seconde et langue maternelle. Elle a collaboré à la rédaction de divers manuels scolaires et ouvrages en éducation, et a participé comme assistante à de nombreuses recherches en didactique, notamment en didactique de l'oral.

Christian Dumais est professeur de didactique du français à 1'Université du Québec à Trois-Rivières (UQTR). Il a fait ses études doctorales en éducation, plus précisément en didactique du français, à l'Université du Québec en Outaouais. Sa thèse porte sur les fondements d'une progression des objets d'enseignement/ apprentissage de l'oral. Il est également titulaire d'une maîtrise en linguistique, profil didactique des langues, de l'Université du Québec à Montréal. Il a été enseignant au primaire et au secondaire à la Commission scolaire de Montréal. Ses travaux de recherche concernent principalement l'enseignement et l'évaluation de l'oral. Il s'intéresse également à la formation des maîtres et au développement des compétences en littératie des élèves du préscolaire au secondaire. Christian Dumais fait partie de l'Équipe de Recherche en Littératie et Inclusion (ÉRLI).

Hélène Meunier est chargée de cours au département d'éducation et pédagogie ainsi qu'au département de didactique à l'Université du Québec à Montréal (UQAM). Elle est aussi conseillère pédagogique à l'UQAM depuis février 2014. Détentrice de deux maîtrises, une en éducation et une en administration scolaire, elle poursuit actuellement des études doctorales en évaluation des apprentissages. Elle s'intéresse à la pédagogie universitaire et à la formation des maîtres. Elle a également été enseignante et directrice d'établissement scolaire au primaire et au secondaire. 


\section{Annexe 1 : Questionnaire de l'enquête}

\section{Questionnaire pour les étudiants de $2^{e}, 3^{e}$ et $4^{e}$ année du baccalauréat}

A. Au cours de votre formation universitaire actuelle, quelles pratiques, évènements ou interventions ont été favorables au développement de votre compétence à l'oral ?

\begin{tabular}{|c|c|c|c|c|c|c|c|}
\hline & $\begin{array}{l}\text { Extrêmement } \\
\text { favorable au } \\
\text { développeme } \\
\text { nt des } \\
\text { compétences } \\
\text { à l'oral }\end{array}$ & 4 & 3 & 2 & 1 & $\begin{array}{l}\text { Extrêmement } \\
\text { défavorable au } \\
\text { développement } \\
\text { des } \\
\text { compétences à } \\
\text { l'oral }\end{array}$ & $\begin{array}{l}\mathrm{Ne} \\
\text { s'applique } \\
\text { pas }\end{array}$ \\
\hline \multicolumn{8}{|l|}{ Contexte universitaire } \\
\hline \multicolumn{8}{|l|}{$\begin{array}{l}\text { Le cours de didactique de } \\
\text { l'oral (DDL 2735) }\end{array}$} \\
\hline \multicolumn{8}{|l|}{$\begin{array}{l}\text { Les cours obligatoires à la } \\
\text { suite d'un échec au test oral } \\
\text { ou suivis par choix (4 } \\
\text { volets LIN1016, LIN1017, } \\
\text { LIN1018, LIN1019): }\end{array}$} \\
\hline \multicolumn{8}{|l|}{$\begin{array}{l}\text { Le cours obligatoire à la suite } \\
\text { d'un échec au test oral ou } \\
\text { suivis par choix ( } 1 \text { volet : } \\
\text { LIN1020) }\end{array}$} \\
\hline \multicolumn{8}{|l|}{$\begin{array}{l}\text { Situation d'apprentissage sur } \\
\text { 1'art de conter (DDM 2500) }\end{array}$} \\
\hline \multicolumn{8}{|l|}{$\begin{array}{l}\text { Le débat éthique (DDM } \\
3550)\end{array}$} \\
\hline \multicolumn{8}{|l|}{$\begin{array}{l}\text { La grille d'autoévaluation et } \\
\text { de coévaluation de l'oral }\end{array}$} \\
\hline \multicolumn{8}{|l|}{$\begin{array}{l}\text { Le site internet « Test de } \\
\text { français oral pour futurs } \\
\text { enseignants » de l'UQAM } \\
\text { http://www.unites.uqam.ca/te } \\
\text { storal/ }\end{array}$} \\
\hline \multicolumn{8}{|l|}{$\begin{array}{l}\text { Le centre d'aide (CARE) et } \\
\text { monitorat }\end{array}$} \\
\hline \multicolumn{8}{|l|}{$\begin{array}{l}\text { Les prestations orales } \\
\text { réalisées dans les cours }\end{array}$} \\
\hline \multicolumn{8}{|l|}{$\begin{array}{l}\text { L'évaluation par les } \\
\text { formateurs des prestations } \\
\text { orales dans les cours }\end{array}$} \\
\hline Les entretiens avec les & & & & & & & \\
\hline
\end{tabular}




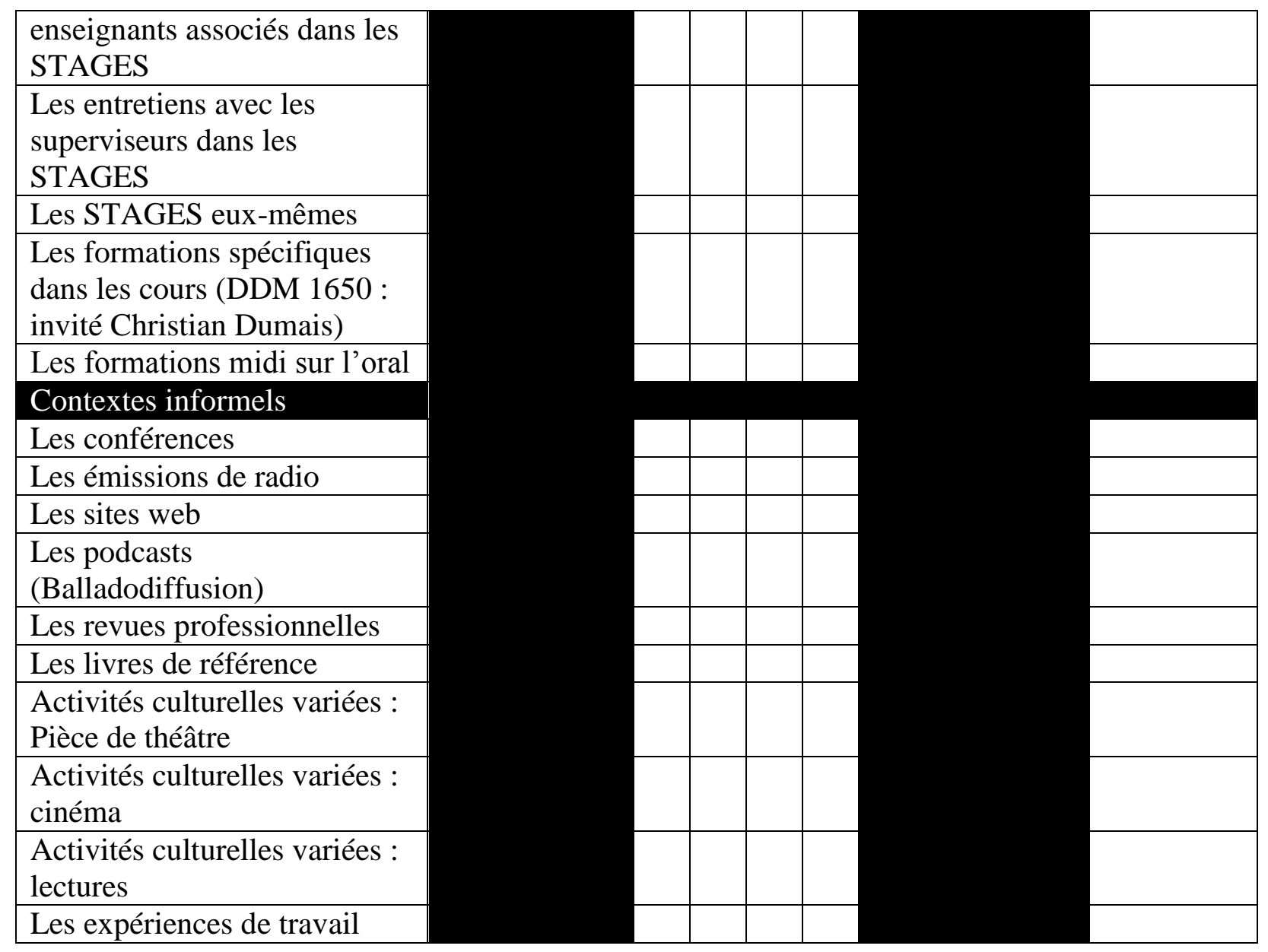

B. Qu'est-ce qui pourrait vous aider davantage?

\begin{tabular}{|c|c|c|c|c|c|c|}
\hline & $\begin{array}{l}\text { Extrêmement } \\
\text { favorable au } \\
\text { développement } \\
\text { des compétences } \\
\text { à l'oral }\end{array}$ & 4 & 3 & 2 & 1 & $\begin{array}{l}\text { Extrêmement } \\
\text { défavorable au } \\
\text { développement } \\
\text { des } \\
\text { compétences à } \\
\text { l'oral }\end{array}$ \\
\hline \multicolumn{7}{|l|}{ Contexte universitaire } \\
\hline \multicolumn{7}{|l|}{$\begin{array}{l}\text { Avoir accès à plus de cours spécialisés } \\
\text { sur l'oral hors programme }\end{array}$} \\
\hline \multicolumn{7}{|l|}{$\begin{array}{l}\text { Que les cours du programme abordent } \\
\text { plus fréquemment l'oral }\end{array}$} \\
\hline \multicolumn{7}{|l|}{$\begin{array}{l}\text { Qu'il y ait une offre de cours } \\
\text { obligatoires plus abondante dans le } \\
\text { programme qui permette de mieux } \\
\text { comprendre l'oral à enseigner }\end{array}$} \\
\hline Qu'il y ait une offre de cours & & & & & & \\
\hline
\end{tabular}


obligatoires plus abondante dans le programme qui permette d'améliorer sa performance à l'oral

Que les superviseurs de stage accordent une plus grande place à l'oral dans l'encadrement des stagiaires.

Que les enseignants associés accordent une plus grande place à l'oral dans l'encadrement des stagiaires.

Que plus d'ateliers d'aide en français liés à la compétence en oral soient disponibles.

Que les ressources web traitant de l'oral offertes à l'université soient plus complètes (le site internet du test oral)

\section{Contextes informels}

Les conférences

Les émissions de radio

Les sites web

Les podcasts (baladodiffusion)

Les revues professionnelles

Les livres de référence

Activités culturelles variées :

Pièce de théâtre

Activités culturelles variées :

cinéma

Activités culturelles variées :

lectures

Les expériences de travail

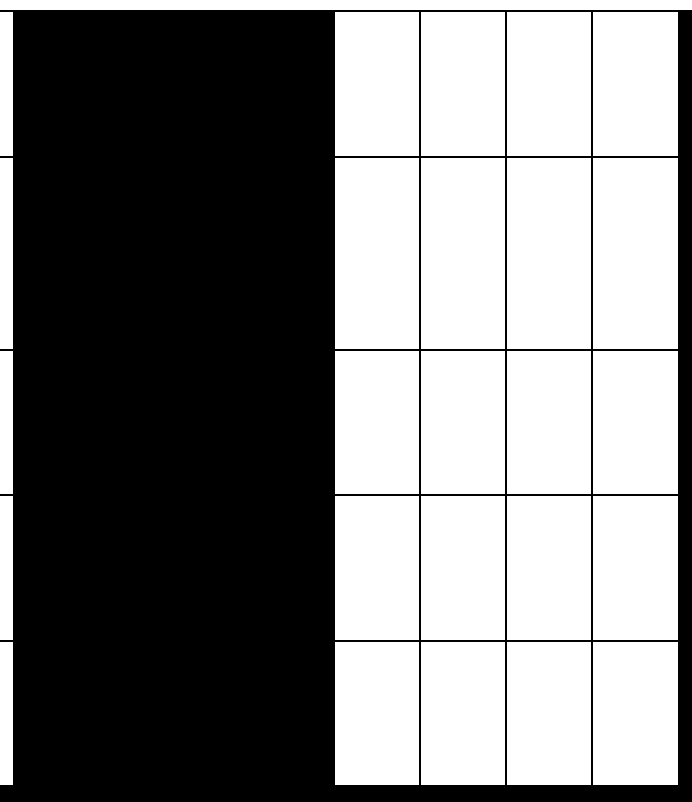

Accepteriez-vous de participer à une entrevue avec un chercheur pour donner suite à vos réponses à ce questionnaire ?

\begin{tabular}{|l|l|}
\hline Oui & \\
\hline Non & \\
\hline
\end{tabular}


A. $\mathrm{Au}$ cours de votre formation antérieure, quelles pratiques, évènements ou interventions ont été favorables au développer de vos compétences à l'oral ?

\begin{tabular}{|c|c|c|c|c|c|c|c|}
\hline & $\begin{array}{l}\text { Extrêmement } \\
\text { favorable au } \\
\text { développement } \\
\text { des } \\
\text { compétences à } \\
\text { l'oral }\end{array}$ & 4 & 3 & 2 & 1 & $\begin{array}{l}\text { Extrêmement } \\
\text { défavorable au } \\
\text { développement } \\
\text { des } \\
\text { compétences à } \\
\text { l'oral }\end{array}$ & $\begin{array}{l}\mathrm{Ne} \\
\text { s'applique } \\
\text { pas }\end{array}$ \\
\hline \multicolumn{8}{|l|}{ Contexte universitaire } \\
\hline \multicolumn{8}{|l|}{$\begin{array}{l}\text { Les cours portant } \\
\text { exclusivement sur l'oral }\end{array}$} \\
\hline \multicolumn{8}{|l|}{$\begin{array}{l}\text { Les sites web concernant l'oral } \\
\text { proposés au cours de votre } \\
\text { formation }\end{array}$} \\
\hline \multicolumn{8}{|l|}{ Les centres d'aide en français } \\
\hline \multicolumn{8}{|l|}{$\begin{array}{l}\text { Les prestations orales réalisées } \\
\text { dans les cours }\end{array}$} \\
\hline \multicolumn{8}{|l|}{$\begin{array}{l}\text { L'évaluation par les } \\
\text { formateurs des prestations } \\
\text { orales dans les cours }\end{array}$} \\
\hline \multicolumn{8}{|l|}{$\begin{array}{l}\text { Les entretiens avec les } \\
\text { enseignants associés dans les } \\
\text { STAGES }\end{array}$} \\
\hline \multicolumn{8}{|l|}{$\begin{array}{l}\text { Les entretiens avec les } \\
\text { superviseurs dans les STAGES }\end{array}$} \\
\hline \multicolumn{8}{|l|}{ Les STAGES eux-mêmes } \\
\hline \multicolumn{8}{|l|}{$\begin{array}{l}\text { Des formations sur l'oral hors } \\
\text { de votre formation antérieure }\end{array}$} \\
\hline \multicolumn{8}{|l|}{ Contextes informels } \\
\hline \multicolumn{8}{|l|}{ Les conférences } \\
\hline \multicolumn{8}{|l|}{ Les émissions de radio } \\
\hline \multicolumn{8}{|l|}{ Les sites web } \\
\hline \multicolumn{8}{|l|}{ Les podcasts (baladodiffusion) } \\
\hline \multicolumn{8}{|l|}{ Les revues professionnelles } \\
\hline \multicolumn{8}{|l|}{ Les livres de référence } \\
\hline \multicolumn{8}{|l|}{$\begin{array}{l}\text { Activités culturelles variées : } \\
\text { Pièce de théâtre }\end{array}$} \\
\hline \multicolumn{8}{|l|}{$\begin{array}{l}\text { Activités culturelles variées : } \\
\text { cinéma }\end{array}$} \\
\hline $\begin{array}{l}\text { Activités culturelles variées : } \\
\text { lectures }\end{array}$ & & & & & & & \\
\hline
\end{tabular}


Les expériences de travail

B. Qu'est-ce qui pourrait vous aider davantage ?

\begin{tabular}{|c|c|c|c|c|c|c|}
\hline & $\begin{array}{l}\text { Extrêmement } \\
\text { favorable au } \\
\text { développement } \\
\text { des compétences } \\
\text { à l'oral }\end{array}$ & 4 & 3 & 2 & 1 & $\begin{array}{l}\text { Extrêmement } \\
\text { défavorable au } \\
\text { développement } \\
\text { des compétences } \\
\text { à l'oral }\end{array}$ \\
\hline \multicolumn{7}{|l|}{ Contexte universitaire } \\
\hline \multicolumn{7}{|l|}{$\begin{array}{l}\text { Des cours spécialisés hors } \\
\text { programme sur l'oral }\end{array}$} \\
\hline \multicolumn{7}{|l|}{$\begin{array}{l}\text { Les cours prévus du programme et } \\
\text { qui portent sur la compétence à l'oral }\end{array}$} \\
\hline \multicolumn{7}{|l|}{ Les stages } \\
\hline \multicolumn{7}{|l|}{ Des ateliers d'aide en français } \\
\hline \multicolumn{7}{|l|}{$\begin{array}{l}\text { Des ressources informatiques } \\
\text { offertes par l'université }\end{array}$} \\
\hline \multicolumn{7}{|l|}{ Contextes informels } \\
\hline \multicolumn{7}{|l|}{ Les conférences } \\
\hline \multicolumn{7}{|l|}{ Les émissions de radio } \\
\hline \multicolumn{7}{|l|}{ Les sites web } \\
\hline \multicolumn{7}{|l|}{ Les podcasts (baladodiffusion) } \\
\hline \multicolumn{7}{|l|}{ Les revues professionnelles } \\
\hline \multicolumn{7}{|l|}{ Les livres de référence } \\
\hline \multicolumn{7}{|l|}{$\begin{array}{l}\text { Activités culturelles variées : } \\
\text { Pièce de théâtre }\end{array}$} \\
\hline \multicolumn{7}{|l|}{$\begin{array}{l}\text { Activités culturelles variées : } \\
\text { cinéma }\end{array}$} \\
\hline \multicolumn{7}{|l|}{$\begin{array}{l}\text { Activités culturelles variées : } \\
\text { lectures }\end{array}$} \\
\hline \multicolumn{7}{|l|}{ Les expériences de travail } \\
\hline \multicolumn{7}{|l|}{ Des activités culturelles } \\
\hline \multicolumn{7}{|l|}{ Des colloques } \\
\hline $\begin{array}{l}\text { Des sites web autres que ceux } \\
\text { proposés par l'université }\end{array}$ & & & & & & \\
\hline
\end{tabular}

Accepteriez-vous de participer à une entrevue avec un chercheur pour donner suite à vos réponses à ce questionnaire ?

\begin{tabular}{|l|l|}
\hline Oui & \\
\hline Non & \\
\hline
\end{tabular}

